\title{
Analyzing Ambiguity in the Standard Definition of Creativity
}

\author{
Thomas R. Colin \\ Plymouth University, UK \\ Thomas.R.Colin@CogNovo.eu \\ Received 17 April 2017; accepted 26 September 2017; published 21 November 2017.
}

\begin{abstract}
The increasingly rich and diverse literature on creativity has its core in psychology, but spans the cognitive sciences from artificial intelligence to philosophy and borrows from the wider humanities. Perhaps because of this immense breadth, there remains considerable disagreement with respect to the identity of the object of research. How to define creativity?

According to the "standard definition," creativity consists of "effectiveness and originality." This definition is (relatively) consensual and therefore appears to capture something common to academic concepts of creativity. I conduct a conceptual analysis of the definition; thereby, I isolate and describe two ambiguities. Firstly, the definition leaves open the choice of the context and norms against which to measure originality and effectiveness. Secondly, it does not discuss the possible role of a subjective judge.

My goal is not to propose yet another model of creativity, but to clearly identify the possible meanings of the word creativity in academic research. The existence of different interpretations does not necessarily reflect a fundamental disagreement about reality, but rather a failure to achieve consensus on a shared technical language. Therefore, simply recognizing and acknowledging the competition between diverse interpretations can form the basis for successful communication and for a complementary division of labor; it could improve the viability of interdisciplinary collaborations and prevent unnecessary fragmentation of the field.
\end{abstract}

Keywords: creativity; definition. 
An incomplete or vague definition is not necessarily damaging, even when it affects the most central concept of a field or discipline. For instance, Trifonov (2011) collected 124 definitions of life (the central concept in biology), and Legg and Hutter (2007) brought together 72 definitions of intelligence (the central concept in artificial intelligence and an important one in psychology). Despite this inability to settle on a definition of their object of study, these fields appear to be thriving. Why, then, should we worry about the definition of creativity? Perhaps because of the disparate roles of these concepts (life, intelligence, creativity) in the corresponding disciplines. Indeed, both "life" in biology and "intelligence" in artificial intelligence are usually relegated to a philosophical backstage; the leading roles are instead given to evolution or medicine (biology), or to planning or learning (artificial intelligence). In contrast, the concept of creativity is at the forefront of creativity research. Creativity researchers often make general claims about the nature of creativity: statements of the form "creativity correlated with $x^{\prime \prime}$ or "creativity requires $y$." If we cannot confidently separate the creative from the non-creative, such statements themselves become vague and ambiguous. To prevent this, we must know what we are talking about when we talk about creativity.

The "standard definition of creativity" (Runco \& Jaeger, 2012) is, in its present form, the fruit of decades of discussions and debates aimed at reaching a point of (approximative) agreement between creativity researchers. The result is a bi-partite definition: creativity requires (1) originality and (2) effectiveness. This definition is best understood by also considering the family of related definitions to which it belongs: "novelty and value" or "novelty and appropriateness."

I propose investigating this definition via conceptual analysis, a philosophical method consisting of clarifying a concept by exploring possible interpretations and testing their internal consistency, sometimes by using thought experiments. The analysis reveals two sources of ambiguity: the relativity of the criteria of originality and effectiveness to a context and norm, and the potential subjectivity of a judge of creativity. I believe these ambiguities, and the misunderstandings they cause, can help explain some of the disagreements and fragmentation in creativity research.

In the next section I discuss the history of the field, introducing some useful distinctions. I then study the standard definition in more detail: what is meant by originality and effectiveness? Finally, I consider some of the implications for creativity research.

\section{Background}

Before discussing the ambiguities of the definition, it is useful to give an overview of past conceptualizations of creativity. ${ }^{1}$

\footnotetext{
${ }^{1}$ For a more detailed account of the story of Creativity Research, interested readers can consult Batey and Furnham (2006) or Hennessey and Amabile (2010). Some of the key characters are cognitive processes, personality traits, social interactions, creative achievements, and practical applications.
} 
The word itself is a surprisingly recent addition to the western vocabulary; ${ }^{2}$ its appearance is almost concomitant with that of Creativity Research as a field, with Guilford's address to the American Psychological Association (Guilford, 1950). Indeed, prior to the 1940s it was virtually unheard of in English, German (Kreativität), or French (créativite). Its popularity rose sharply and steadily until the 2000s (Google Books Ngram Viewer, 2017); the European Union branded 2009 the "Year of Creativity and Innovation" (European Commission, 2009). As the popularity of the word grew, academics devised models of creativity. Important such models include:

- Stage-based models (Wallas, 1926): the creative process is divided into separate stages, such as preparation, incubation, illumination, and verification. Later models (see Lubart, 2001) have included stages for problem-finding or for the communication of results.

- Convergent and Divergent thinking (Guilford, 1956): creativity is considered within a theory of the intellect, in which creativity results from the interplay of convergent and divergent productive processes.

- Blind Variation, Selective Retention (BVSR; Campbell, 1960): this recently revitalized (Simonton, 2011) model focuses on a trial-and-error explanatory framework; it is amenable to cognitive/computational as well as social interpretations.

- The Systems Model (Csikszentmihalyi, 1996, p. 6): “creativity results from the interaction of a system composed of three elements: a culture that contains symbolic rules, a person who brings novelty into the symbolic domain, and a field of experts who recognize and validate the innovation."

In order to handle the concepts presented in different explanatory models, various taxonomies have been proposed. Such work includes the "four Cs" (Kaufman \& Beghetto, 2009), which distinguish types of creativity ranging from "mini-c" (creativity in learning and development) to "big-C" (eminent creativity). Another example is the four Ps framework (Rhodes, 1961), which refers to the elements of creativity research as the Person, Product (object, idea, behavior...), Process, and Press (interactions with the social environment of the creator).

These variegated views and frameworks illustrate the fragmentation of the field (recognized with concern in, e.g., Hennessey \& Amabile, 2010). This fragmentation is also evident in comprehensive introductions to the field, such as Sawyer (2012) or Runco (2014), in which different sections or chapters discuss cognitive, developmental, neurobiological, social, educational, and cultural perspectives ... among others.

\footnotetext{
${ }^{2}$ However, related terms and concepts predate it; for instance, there was considerable discussion of genius in the $19^{\text {th }}$ century.
} 
Indeed, creativity research has produced an awe-inspiring variety of "perspectives" and "approaches," but it has failed to converge towards a big picture. I believe this is because the researchers share the same lexicon, but often do not speak the same language.

In light of this history, it should come as no surprise that the word creativity is fought over by academic communities who attach different meanings to it. But a word with multiple competing meanings is impractical for communication. How can misunderstandings be avoided? A radical solution would be to stop using the term altogether in favor of a set of more precise alternatives; this is unrealistic due to the cachet that creativity already has. Therefore, I suggest a more pragmatic approach: let us identify and list the different meanings attached to creativity and use this list to disambiguate the uses of the term in creativity research. In this article, the first step of this program takes the form of a conceptual analysis.

\section{Conceptual Analysis of the Standard Definition}

Margolis and Laurence (2014) define conceptual analysis as "a distinctively a priori activity that many take to be the essence of philosophy ... Paradigmatic conceptual analyses offer definitions of concepts that are to be tested against potential counterexamples that are identified via thought experiments" (§ 2.1, para. 3). Conceptual analysis has been criticized (Margolis \& Laurence, 2014) on the grounds that the intuitions with which we navigate thought experiments can be individual- or culturedependent. In an attempt to contain this risk, I will not consider the concepts of creativity used by lay people or by creatives: I expect these to be sometimes incoherent, and usually inconsistent across individuals, communities, or cultures. Instead, I focus on the definitions and intuitions put forward by creativity researchers whose views are informed by experimental evidence, correspond to an extensive knowledge of the field, and aim to achieve consensus.

\section{The Standard Definition}

The "standard definition," summarized by Runco and Jaeger (2012) as "originality and effectiveness" (p. 92), is related to a cluster of bi-partite definitions which has accumulated since the origin of the field. Each component is better understood by considering the cluster of related terms: ${ }^{3}$

- Originality, Novelty/Novel/New, Non-obvious, Uncommon, Unique.

- Effectiveness, Adaptive, Appropriateness, Correct, Fit, Good, Realistic and acceptable, Relevant, Valuable, Usefulness, Worthwhile and compelling.

\footnotetext{
${ }^{3}$ Runco and Jaeger (2012) provide references for the corresponding definitions.
} 
Unfortunately, these definitions make use of concepts that are themselves underspecified. This is no coincidence: they harness ambiguous terms to describe an ambiguous concept. Instead of decomposing creativity into more precise and fundamental concepts (as an explanatory definition would: "water is $\mathrm{H}_{2} \mathrm{O}$ "), some vagueness is preserved. This could be by design: to be consensual, the definition must preserve the existing inconsistencies and disagreement between researchers. There is only one way to preserve these disagreements in a definition while avoiding incoherence and contradiction: the definition must be ambiguous, under-specified. Indeed, this is what makes this definition an interesting starting point for elucidating ambiguity in the word creativity.

What ambiguity is there? I find two different sorts.

\section{First Ambiguity: Context and Norm}

The first sort is the relativity of the two components. Absolute interpretations are not taken seriously in the field: that is, few believe that creativity requires either absolute novelty or originality, or objective good. Thus, originality and effectiveness must be relative to something. But relative to what?

1. Originality is relative to a context: originality can only be measured relative to a group, and novelty relative to a history. I will refer to this group or history as the context.

2. Effectiveness is relative to a norm: effectiveness, goodness, relevance, can only be measured with respect to goals, criteria, or values; in the most general sense these are called norms.

In practice, creativity research often lets context and norm vary together by specifying them based on a singular point of view. For instance, one may implicitly choose the point of view of the creator: the creative product must be new to the creator (context), and effective for the creator's purposes (norm). It is also possible to prefer some group external to the creator: the product must then be original within the group (context), and effective for the group (norm).

Thus, the concise definition, "creativity requires both originality and effectiveness," can be rephrased as "creativity with respect to a context and a norm requires both effectiveness relative to the given norm and originality relative to the given context." This more cumbersome definition makes it explicit that the components (originality and norm) are ambiguous whenever norm and context are not specified.

To illustrate this point, consider the following two examples of creative individuals drawn from distant branches of the literature: the New Caledonian crow Betty, and the $20^{\text {th }}$ century post-impressionist painter Vincent van Gogh. Betty was dubbed creative (Weir \& Kacelnik, 2006) for building a tool to solve a problem. The crow had 
never made such a tool before, and had not seen another crow make one, hence the tool-building process was original within Betty's context and was effective at reaching the food reward. Contrast with Van Gogh, whose widely recognized creativity is dependent not merely on the novelty of his paintings with respect to his personal history, nor to their fit with his own norm of artistic value. Instead, the creativity of the painter is measured against the history of art, the artists of his time, and the norms of today's art connoisseurs and art historians. Based on the definition, we are justified in saying that both Betty and Van Gogh are creative in some sense; but they are creative relative to different contexts and norms. The difference between them is not (just) quantitative, but qualitative because different norms and contexts and therefore different concepts of creativity are involved.

\section{Second Ambiguity: The Interplay between Creator and Judge}

The second source of ambiguity is the person or group emitting the creativity judgement, presumably based on the two criteria. This is surprising: while in the previous section I described creativity as relative (to norms and context), it remained nevertheless possible to directly measure it against these elements, if they were supplied. But a subjective judge is now introduced, typically a community or even a field. This negates the possibility of assessing the creativity of an individual in isolation; this undermines branches of creativity research that focus on cognitive characteristics at the individual level.

But this is what major figures of creativity research have done. For instance, Runco and Jaeger (2012) observe that "The standard definition only pinpoints which criteria must be used; it does not say anything about who is to judge each" (p. 92) Csikszentmihalyi (1996, pp. 23-25) explicitly asks his readers to pick a judge: either the creators themselves, or relevant members of society, the latter having his vote. Later, he presents the following thought experiment: “[Van Gogh's creativity] came into being when a sufficient number of art experts felt that his paintings had something important to contribute" (Csikszentmihalyi, 1996, p. 31). For Csikszentmihalyi, Van Gogh therefore became creative after his death, by virtue of the changing opinions of art experts.

Why would the terms of my first distinction not suffice? Distinguishing different contexts and norms, one could write that Van Gogh was both (1) not creative based on the criteria of a first group (his contemporaries), but (2) was creative relative to the criteria of another group (later critics). However, these authors rejected this path. I surmise that, for them, creativity involves a dynamic interaction between the creator and the judges, such that the creator is able to alter the norms by which the product is evaluated. This is most clear in the case of the arts, and perhaps best expressed by Proust (2006/1921) with respect to another painter:

People of taste and refinement tell us nowadays that Renoir is one of the great painters of the last century. But in so saying they forget ... that it took a great deal of time, well into the present century, before Renoir was hailed as a great artist. To 
succeed thus in gaining recognition, the original painter, the original writer proceeds like an oculist. The course of treatment they give us by their painting or by their prose is not always agreeable to us. When it is at an end the operator says to us: "Now look!" And, lo and behold, the world around us (which was not created once and for all, but is created afresh as often as an original artist is born) appears to us entirely different from the old world, but perfectly clear. Women pass in the street, different from what they used to be, because they are Renoirs, those Renoir types which we persistently refused to see as women. The carriages, too, are Renoirs, and the water, and the sky. (p. 1131)

The creator, via his creation, proceeds "like an oculist": transforming the vision of the judges, and thereby, their judgement. Is this specific to the arts? Kuhn (1970) sees a similar phenomenon in the sciences, in which an "incommensurable" innovation must lead to the "conversion" of a scientific community to new criteria of good science. In this view, creativity is an emergent social phenomenon rather than a mere cognitive ability.

However, this interpretation seems rather more applicable to the eminent, "big-C" creativity of historical importance, than to the "mini-C" or "little-C" creativity of children and daily life. Indeed, Csikszentmihalyi's Van Gogh thought experiment could "seem insane" to some readers, such as, perhaps, experimental psychologists attempting to measure creativity in the lab. It is more useful for those focused on the process of creative thought, for instance the insight phenomenon, to adopt a more prosaic interpretation of the definition, according to which Betty the crow is creative.

\section{Discussion}

I have presented two types of ambiguity in the definition of creativity. The first concerns the context and norm against which originality and effectiveness are to be measured. The second concerns the existence and identity of a judge of creativity.

The use of a definition with multiple interpretations might correspond to a belief that the different types of creativity are, if not identical, at least related. For instance, the first ambiguity can be set aside when the norms and context of the individual and those of society are close enough to produce similar evaluations of creativity. The second ambiguity, despite considering creativity as an emergent social phenomenon, nonetheless suggests the involvement of certain cognitive processes on the part of the creator. In particular, there is a striking parallel between the "paradigm shifts" (Kuhn, 1970) seen in the most eminent of scientific or artistic works and the representational change observed in insight problem-solving (Ohlsson, 1992). The consensus view is that, despite the differences, the areas of agreement justify the unity of the field (Tardif \& Sternberg, 1988). Despite this relatedness, it is not difficult to provide examples which satisfy one interpretation of the definition, but fail to satisfy another; I have done so in the previous section. This justifies my recommendation to explicitly state the interpretation of the definition whenever the word creativity is used. 
How might these ambiguities affect the field? Consider, for example, the muchdebated relationship between mental illness and creativity (e.g., Kyaga et al., 2011). Mental illness causes ineffective behavior relative to oneself, but exploring the lesstraveled path may increase the probability of discovering something valuable relative to the community. Therefore, differing interpretations of creativity with respect to the first ambiguity may help explain the heated disagreement on this issue.

Let us consider another example in the sub-field of computational creativity. The interpretation of creativity in Wiggins (2006) focuses on cognitive processes and therefore appears to fit best with an interpretation in terms of originality and effectiveness relative to the agent's point of view. In contrast, the interpretation in Colton, Charnley and Pease (2011) explicitly considers interactions with a spectator, thus allowing for the presence of a judge. The two interpretations are apparently conflicting, but they can be viewed as complementary in light of this analysis.

Ultimately, a definition is a matter of convention, not of fact. Being aware of possible ambiguities allows the intended interpretation to be clearly specified, and this can suffice to restore shared understanding.

\section{Conclusion}

I have analyzed the standard definition of creativity in terms of its interpretation in creativity research. Doing so, I found two sources of ambiguity. The first is the relativity of creativity to a particular context and set of norms, against which one can measure originality and effectiveness. The second is the introduction, or not, of a subjective judge, whose norms for judgement can be influenced.

Sawyer (2012) views creativity research as divided and claims that as long as the different communities proceed "on separate tracks, we will fail to explain creativity" (p. 14). By studying the definition of creativity, I hope to have shed light on hidden causes for these divisions; creativity researchers study different concepts (creativity relative to oneself, to a group; involving a subjective judge or not), but collectively refer to these concepts using the same label. The recognition of these semantic differences could be an important step towards successful collaboration.

The work conducted here is philosophical, but it could be (in part) empirically tested. My analysis makes testable predictions about what academics believe: if the analysis is correct, it must be possible to find semantic clusters in the creativity literature corresponding to the different interpretations of the word creativity. This could be done by adapting the method of Jordanous and Keller (2016). 


\section{Acknowledgements}

This work was completed as part of the Marie Curie Initial Training Network FP7PEOPLE-2013-ITN, CogNovo, grant number 604764, in which 25 doctoral candidates from different disciplines worked on topics related to creativity and cognitive innovation. I would like to thank Mona Nasser, Tony Belpaeme, and especially Frank Loesche for their helpful remarks.

\section{References}

Batey, M., \& Furnham, A. (2006). Creativity, intelligence, and personality: A critical review of the scattered literature. Genetic, social, and general psychology monographs, 132(4), 355-429. doi:10.3200/MONO.132.4.355-430

Campbell, D. T. (1960). Blind variation and selective retentions in creative thought as in other knowledge processes. Psychological Review, 67(6), 380-400. doi:10.1037/h0040373

Colton, S., Charnley, J., \& Pease, A. (2011). Computational creativity theory: The FACE and IDEA descriptive models. In D. Ventrua, P. Gervás, D. F. Harrell, M. L. Maher, A. Pease, \& G. Wiggins (Eds.), Proceedings of the Second International Conference on Computational Creativity (pp. 90-95). Mexico City, Mexico: UAM Cuajimalpa.

Csikszentmihalyi, M. (1996). Creativity: Flow and the psychology of discovery and invention. New York, NY: HarperCollins.

European Commission. (2009). European year of creativity and innovation 2009. Retrieved from http://www.create2009.europa.eu

Google Books Ngram Viewer. (2017). Google Books Ngram Viewer. Retrieved from http://books.google.com/ngrams

Guilford, J. P. (1950). Creativity. American Psychologist, 5(9), 444-454.

Guilford, J. P. (1956). The structure of intellect. Psychological Bulletin, 53(4), 267-293. doi:10.1037/h0040755

Hennessey, B. A., \& Amabile, T. M. (2010). Creativity. Annual Review of Psychology, 61(1), 569-598. doi:10.1146/annurev.psych.093008.100416

Jordanous, A., \& Keller, B. (2016). Modelling creativity: Identifying key components through a corpus-based approach. PLoS ONE, 11(10), e0162959. doi:10.1371/journal.pone.0162959

Kaufman, J. C., \& Beghetto, R. A. (2009). Beyond big and little: The four c model of creativity. Review of General Psychology, 13(1), 1-12. doi:10.1037/a0013688

Kuhn, T. S. (1970). The structure of scientific revolutions. Chicago, IL: University of Chicago Press.

Kyaga, S., Lichtenstein, P., Boman, M., Hultman, C., Långström, N., \& Landén, M. (2011). Creativity and mental disorder: Family study of 300000 people with severe mental disorder. The British Journal of Psychiatry, 199(5), 373-379. doi:10.1192/bjp.bp.110.085316

Legg, S., \& Hutter, M. (2007). A collection of definitions of intelligence. Frontiers in Artificial Intelligence and Applications, 157, 17-24. 
Lubart, T. I. (2001). Models of the creative process: Past, present and future. Creativity Research Journal, 13(3-4), 295-308. doi:10.1207/S15326934CRJ1334_07

Margolis, E. \& Laurence, S. (2014). Concepts. In E. N. Zalta (Ed.), The Stanford encyclopedia of philosophy (Spring 2014 Edition). Standord, CA: Metaphysics Research Lab, Stanford University. Retrieved from https://plato.stanford.edu/archives/spr2014/entries/concepts/

Ohlsson, S. (1992). Information-processing explanations of insight and related phenomena. In M. Keane \& K. Gilhooey (Eds.), Advances in the psychology of thinking (pp. 1-44). London, UK: Harvester-Wheatsheaf.

Proust, M. (2006). Remembrance of things past: Volume one. (C. K. Scott Moncrieff, Trans.). Wordworth Editions: Ware, UK. (Original work published 1921)

Rhodes, M. (1961). An analysis of creativity. The Phi Delta Kappan, 42(7), 305-310. doi: $10.2307 / 20342603$

Runco, M. A. (Ed.). (2014). Creativity (2nd ed.). San Diego, CA: Academic Press. doi:10.1016/B9780-12-410512-6.00014-X

Runco, M. A. \& Jaeger, G. J. (2012). The standard definition of creativity. Creativity Research Journal, 24(1), 92-96. doi:10.1080/10400419.2012.650092

Sawyer, R. K. (2012). Explaining creativity: The science of human innovation (2nd ed.). Oxford, UK: Oxford University Press.

Simonton, D. K. (2011). Creativity and discovery as blind variation: Campbell's (1960) BVSR model after the half-century mark. Review of General Psychology, 15(2), 158-174. doi:10.1037/a0022912

Tardif, T. Z., \& Sternberg, R. J. (1988). What do we know about creativity? In R. J. Sternberg (Ed.) The nature of creativity: Contemporary psychological perspectives (pp. 429-440). New York, NY: Cambridge University Press.

Trifonov, E. N. (2011). Vocabulary of definitions of life suggests a definition. Journal of Biomolecular Structure and Dynamics, 29(2), 259-266. doi:10.1080/073911011010524992

Wallas, G. (1926). The art of thought. New York, NY: Harcourt, Brace and Co.

Weir, A. A., \& Kacelnik, A. (2006). A New Caledonian crow (Corvus moneduloides) creatively re-designs tools by bending or unbending aluminium strips. Animal cognition, 9(4), 317-334. doi:10.1007/s10071-006-0052-5

Wiggins, G. A. (2006). Searching for computational creativity. New Generation Computing, 24(3), 209-222. 